\title{
The relation between pre-eclampsia at term and neonatal encephalopathy
}

\author{
L Impey, C Greenwood, O Sheil, K MacQuillan, M Reynolds, C Redman
}

\begin{abstract}
Objectives-To determine whether preeclampsia, hypothesised to be an inflammatory condition, is associated with fever in term labour, and confirm and examine the reported association of pre-eclampsia at term with neonatal encephalopathy.

Design-Prospective cohort study.

Setting-A Dublin teaching hospital.

Participants-6163 women in labour with singleton pregnancies at term at low risk for intrapartum hypoxia, recruited to a randomised trial examining the effect of admission cardiotocography on neonatal outcome.
\end{abstract}

Results-Pre-eclampsia was associated with maternal fever $>37.5^{\circ}$ in labour (odds ratio (OR) $3.39,95 \%$ confidence interval (CI) 2.1 to 5.4); this was independent of obstetric intervention (adjusted OR $2.07,95 \%$ CI 1.24 to 3.47 ). Pre-eclampsia was associated with neonatal encephalopathy (OR 25.5, 95\% CI 8.4 to 74.7 ); this too was independent of obstetric intervention (adjusted OR $18.5,95 \%$ CI 5.9 to 58.1 ). Cord arterial $\mathrm{pH}$ values were significantly lower in pre-eclamptics (7.20 $v$ 7.24), although severe cord acidaemia was not significantly more common (OR $2.91,95 \%$ CI 0.7 to 9.9). The association of preeclampsia with encephalopathy was independent of maternal fever (adjusted OR $16.5,95 \%$ CI 5.1 to 54 ) and cord acidaemia (adjusted OR 13.5, 95\% CI 3.2 to 56.7).

Conclusions-The association of preeclampsia with maternal fever at term supports the hypothesis that preeclampsia is an inflammatory condition. The association of pre-eclampsia with neonatal encephalopathy is independent of obstetric intervention and cannot be explained by either acidaemia or maternal fever. A systemic inflammatory response in the fetus, perhaps secondary to oxidative stress, could explain the link between maternal pre-eclampsia and neonatal encephalopathy, and this may occur through cerebral vasoconstriction.

(Arch Dis Child Fetal Neonatal Ed 2001;85:F170-F172)

Keywords: pre-eclampsia; encephalopathy; fever; labour

Nuffield Department of Obstetrics and Gynaecology,

University of Oxford,

John Radcliffe Hospital

C Redman

Correspondence to: Mr Impey, Level 4, The Women's Centre, John Radcliffe Hospital, Headley Way, Headington, Oxford OX3 9DU, UK

Lawrence.Impey@orh.nhs.uk

Accepted 21 June 2001 absence of evidence of infection ${ }^{3}$, and autoimmune disease may also predispose to cerebral palsy, ${ }^{5}$ the association of an inflammatory state with adverse neonatal outcome is not entirely attributable to infection.

We have provided evidence that preeclampsia results from exaggeration of a maternal systemic inflammatory response common to all pregnancies. ${ }^{6}$ The theory is consistent with many of the clinical observations and associations of pre-eclampsia, yet fever, a common manifestation of an inflammatory response, is not considered to be a feature of pre-eclampsia. The hypothesis would predict that pre-eclamptic women would at least be more susceptible to fever. This may be most easily detected in labour, but has not been studied.

We therefore determined whether preeclampsia at term is associated with maternal fever in labour. When we found that it is, we investigated the association between preeclampsia at term and neonatal encephalopathy and analysed whether this is associated with maternal fever.

\section{Methods}

The data were prospectively collected from a cohort of women recruited from August 1997 to April 2000 at a Dublin teaching hospital to a randomised, controlled trial determining the effect of admission cardiotocography on the incidence of adverse neonatal outcome. Women with a singleton fetus were eligible if clear liquor was detected at early amniotomy and the fetus was not considered at risk for intrapartum fetal distress - for example, known intrauterine growth restriction, antepartum haemorrhage. In addition, women with a breech presentation, those delivering before 37 and after 42 completed weeks, and those whose babies were anomalous or had inborn errors of metabolism were excluded from analysis.

Pre-eclampsia was defined as new hypertension in pregnancy ( $\geqslant 140 / 90)$ with new proteinuria (1+ or more on dipstick testing). Maternal pyrexia in labour was an oral temperature $>37.5^{\circ} \mathrm{C}$. Severe acidaemia was defined as a cord arterial $\mathrm{pH}<7.00$ with a base deficit $>12 \mathrm{mmol} / 1$. C reactive protein levels and blood cultures were examined in encephalopathic babies only. Neonatal encephalopathy was diagnosed as described by Sarnat and Sarnat $^{7}$ (grade 2-3 only). The occurrence of cerebral palsy was not calculated, as our infants are not yet old enough to exclude this diagnosis.

Data were analysed using SPSS 9.0 (Chicago, Illinois, USA) and Epi-Info, and crude odds ratios (ORs) were calculated. Adjusted 
Table 1 Associations of pre-eclampsia in term neonates

\begin{tabular}{lllll}
\hline Outcome & $\begin{array}{l}\text { Pre-eclampsia } \\
n(\%)\end{array}$ & $\begin{array}{l}\text { No pre-eclampsia } \\
n(\%)\end{array}$ & $\begin{array}{l}\text { Crude odds ratio } \\
\text { (95\% CI) }\end{array}$ & $\begin{array}{l}\text { Adjusted odds ratio } \\
\text { (95\% CI) }\end{array}$ \\
\hline Total & $124(2.0)$ & $6039(98.0)$ & $3.39(2.10$ to 5.42$)$ & $2.07(1.24 \text { to } 3.47)^{\star}$ \\
Fever $>37.5^{\circ} \mathrm{C}$ & $25(20.2)$ & $419(6.9)$ & $2.91(0.72$ to 9.85$)$ & \\
Severe acidaemia & $3(2.4)$ & $51(0.84)$ & $25.5(8.39$ to 74.7$)$ & $18.50(5.92$ to 58.13$) \dagger$ \\
Encephalopathy & $6(4.8)$ & $12(0.20)$ & & $16.56(5.07$ to 54.03$) \ddagger$ \\
& & & & $13.48(3.21$ to 56.70$) \S$
\end{tabular}

^Adjusted for parity, birth weight, gestation, induction of labour, length of labour, epidural analgesia, and use of oxytocin.

†Adjusted for parity, birth weight, gestation, induction of labour, length of labour, epidural analgesia, use of oxytocin, and instrumental delivery.

$\ddagger$ Adjusted, in addition, for maternal fever in labour $>37.5^{\circ} \mathrm{C}$.

\Adjusted, in addition, for cord arterial $\mathrm{pH}$.

Table 2 Relation between cord arterial pH/base excess and pre-eclampsia in term neonates

\begin{tabular}{|c|c|c|c|c|}
\hline \multirow[b]{2}{*}{ Outcome } & \multicolumn{2}{|l|}{ All babies } & \multicolumn{2}{|c|}{ Encephalopathic babies } \\
\hline & Pre-eclampsia & No pre-eclampsia & Pre-eclampsia & No pre-eclampsia \\
\hline \multicolumn{5}{|l|}{$\mathrm{pH}$} \\
\hline $\mathrm{n}^{\star}$ & 116 & 5634 & 6 & 12 \\
\hline mean & 7.196 & $(\mathrm{p}<0.001)$ & 7.123 & $(\mathrm{p}=0.54)$ \\
\hline range & $6.82-7.40$ & $6.77-7.53$ & $6.82-7.29$ & $6.83-7.33$ \\
\hline \multicolumn{5}{|l|}{ Base deficit } \\
\hline $\mathrm{n}^{\star}$ & 91 & 5042 & 3 & 7 \\
\hline mean & 8.826 & $(\mathrm{p}=0.005)$ & 7.800 & $(\mathrm{p}=0.03)$ \\
\hline range & $19.8-1.5$ & $31.0-1.2$ & $10.8-6.20$ & $21.0-8.20$ \\
\hline
\end{tabular}

${ }^{\star}$ Cord blood gases were not measured in all babies; base deficit measurements were more often missing.

ORs were calculated using multiple logistic regression to examine whether the association of pre-eclampsia with both encephalopathy and maternal fever was independent of factors associated with pre-eclampsia that could be determinants of the outcome. In addition, when investigating the association of preeclampsia with neonatal encephalopathy, we adjusted for both maternal fever and cord gas acidaemia separately, to ascertain their roles in the causal pathway.

\section{Results}

Of 6163 women in labour who met the study criteria, $124(2.0 \%)$ had pre-eclampsia; labour was induced in 87 of these $(70.2 \%)$. Intrapartum pyrexia was recorded in $419(6.9 \%)$; severe acidaemia occurred in $54(0.9 \%)$, and neonatal encephalopathy in $18(0.3 \%)$. Among the encephalopathic neonates, one born to a non-pre-eclamptic mother had raised $C$ reactive protein and positive blood cultures; another born to a pre-eclamptic mother had slightly increased $\mathrm{C}$ reactive protein only.

Pre-eclampsia was associated with shorter gestation, induction of labour, lower parity, lower birth weight, epidural analgesia, longer labour, use of oxytocin, and instrumental delivery.

Neonatal encephalopathy was associated with pre-eclampsia; this was independent of these variables (table 1). Maternal pyrexia was also significantly associated with pre-eclampsia (table 1): this was also independent of the above covariates (instrumental delivery was not analysed as it could not cause intrapartum fever).

Cord gas values were obtained in 116 (94\%) of pre-eclamptic deliveries and 5634 (93\%) of non-pre-eclamptic deliveries $(p=0.51)$. Severe acidaemia was significantly associated with encephalopathy (OR 63.5, 95\% confidence interval (CI) 20.3 to 192). The mean cord arterial $\mathrm{pH}$ after pre-eclamptic pregnancies was significantly lower, and the base deficit significantly higher, than among other babies (table 2). However, the association between severe acidaemia and pre-eclampsia (table 1) did not reach statistical significance. Among the encephalopathic babies, there was a trend towards a higher $\mathrm{pH}$ and lower base deficit in those born to mothers with pre-eclampsia (table 2).

The association of pre-eclampsia with neonatal encephalopathy was independent of maternal fever and umbilical cord acidaemia (table 1).

\section{Discussion}

Our finding that pre-eclampsia is an independent risk factor for maternal fever in labour is consistent with the hypothesis that preeclampsia is itself a systemic inflammatory state. $^{6}$

The association between term pre-eclampsia and neonatal encephalopathy, from which more than half the affected babies die or develop severe disability, ${ }^{8}$ confirms previous case-control studies. ${ }^{1}$ Indeed, one third of our encephalopathic babies followed pregnancies complicated by pre-eclampsia, as do a significant proportion of cases of cerebral palsy. ${ }^{9}$ In addition, we showed that this relation is not the result of obstetric intervention such as induction, epidural analgesia, or augmentation. The lower mean cord arterial $\mathrm{pH}$ and higher base deficits in pre-eclamptic pregnancies were compatible with relative hypoxaemia. Yet the risk of encephalopathy was independent of cord acidaemia. Indeed, there was a trend towards less acidaemia in encephalopathic neonates delivered to pre-eclamptic women.

These data are consistent with the consensus that neonatal encephalopathy does not depend on severe intrauterine hypoxaemia. They reinforce the belief that antepartum factors are important in the development of neonatal encephalopathy ${ }^{1}$ and cerebral palsy. ${ }^{8}$ However, the mechanism by which antepartum factors such as pre-eclampsia lead to encephalopathy remains unexplained.

The association of pre-eclampsia with maternal fever, itself a risk factor for encephalopathy, ${ }^{14}$ could not entirely explain the increased risk of encephalopathy in this group of pre-eclamptic women. Hence it is likely that fever itself is not causal, and that some other feature of the disease leads to the adverse neonatal outcome. There is compelling evidence of a role for perinatal inflammatory processes not exclusive to infection in the development of long term neonatal handicap. ${ }^{5}$ It is therefore relevant that there is an increased systemic inflammatory response in the newborns of preeclamptic women. ${ }^{10}{ }^{11}$ Whether this is linked in some way to the maternal inflammatory response or has a separate cause is not known.

The origin of the fetal inflammatory response in pre-eclampsia is likely to be hypoxia and oxidative stress. These are well recognised 
stimuli to localised ${ }^{12}$ and systemic ${ }^{13}$ inflammatory responses. The fetuses of pre-eclamptic women may be hypoxaemic, which is consistent with our finding of a greater mean base deficit and lower mean $\mathrm{pH}$. Such hypoxaemia is reflected in increased markers of oxidative stress in the placenta ${ }^{14}$ and fetal blood ${ }^{15}$ in preeclampsia and is considered to result from poor uteroplacental perfusion. ${ }^{16}$

Extreme inflammatory responses lead to vasodilatation and shock as in sepsis. However, vasoconstriction is characteristic of less extreme inflammatory stimuli ${ }^{17}$ which could jeopardise tissue perfusion locally. The fetal and neonatal cerebral circulations may be particularly vulnerable, explaining the rare association of pre-eclampsia with neonatal cerebral, particularly parietal lobe, infarcts. ${ }^{18}$ Labour may be a complicating factor. Normal spontaneous labour at term is associated with an increased systemic inflammatory response of the newborn. ${ }^{19}$ It causes fetal head compression, ${ }^{20}$ which, even in uncomplicated labour, is associated with disturbed indices of middle cerebral artery flow $^{21}$ and localised hypoxic changes. ${ }^{22}$ Hence antenatal risk factors may be aggravated by labour, a possibility consistent with the observation that elective caesarean section protects against neonatal encephalopathy. ${ }^{23}$ Whether labour is implicated or not, localised cerebral vasoconstriction, hypoxia, and neuronal damage could occur. The key point is that this need not be in the context of global asphyxia nor even reflected in changes in umbilical blood gas analyses.

An inflammatory response appears to be a common pathway for infection and autoimmune disease in the development of neurological handicap. We propose that it is also a pathway for pre-eclampsia, and that systemic inflammation leads to localised cerebral ischaemia. Future research could address indices of perinatal systemic inflammation rather than gas analysis in cord blood, and relate the results of fetal and neonatal brain imaging to these and antenatal risk factors.

We are indebted to the midwives and women of the National Maternity Hospital, Dublin, and are grateful to Dr Mary Anthony and Dr Peter Brocklehurst for their help in the preparation of this paper. The funding source was The National Maternity Hospital Research Fund. There were no conflicts of interest.

Authors' contributions: LI designed the study, performed statistical analysis, and wrote the paper. OS was principal grant holder, and contributed to the study design and the final draft of the paper. CR and CG contributed to the study design, analysis, and cowrote the paper. MR and KMcQ collected the data and contributed to the final draft of the paper.

1 Badawi N, Kurinczuk J, Keogh JM, et al. Antepartum risk factors for newborn encephalopathy: the Western Australfactors for newborn encephalopathy: the Wester
ian case-control study. BMF 1998;317:1549-53.

2 Gaffney G, Squier M, Johnson A, et al. Clinical associations of prenatal ischaemic white matter injury. Arch Dis Child Fetal Neonatal Ed 1994;70:F101-6.

3 Grether J, Nelson K. Maternal infection and cerebral palsy in infants of normal birth weight. $\mathcal{F} A M A 1997 ; 278: 207-$ 11.

4 Impey L, Greenwood C, MacQuillan K, et al. Fever in labour and neonatal encephalopathy: a prospective cohort study. Br f Obstet Gynaecol 2001;108:594-7.

5 Nelson KB, Dambrosia JM, Grether JK, et al. Neonatal cytokines and coagulation factors in children with cerebral palsy. Ann Neurol 1998;44:665-75.

6 Redman CWG, Sacks GP, Sargeant IL. Pre-eclampsia: an excessive maternal inflammatory response to pregnancy. Am f Obstet Gynecol 1999;180:499-506.

7 Sarnat HB, Sarnat MS. Neonatal encephalopathy following fetal distress. Arch Neurol 1976;33:696-705.

8 Ellenberg JH, Nelson KB. Cluster of perinatal events identifying infants at high risk for death or disability. $\mathcal{F}$ Pediatr fying infants at high

9 Gaffney G, Flavell V, Johnson A, et al. Cerebral palsy and neonatal encephalopathy. Arch Dis Child Fetal Neonatal Ed 1994;70:F195-200

10 Mellembakken J, Aukrust P, Ueland T, et al. Granulocytes and monocytes are activated in the fetal circulation in preeclampsia. Hypertension in Pregnancy 2000;19(suppl 1): 133 .

11 Davidge ST, Signorella AP, Lykins DL, et al. Evidence of endothelial activation and endothelial activators in cord blood of infants of pre eclamptic women. Am 7 Obstet Gynecol 1996;175:1301-6.

12 Michiels C, Arnould T, Remacle J. Endothelial cell responses to hypoxia: initiation of a cascade of cellular interactions. Biochim Biophys Acta 2000;1497:1-10.

13 Hartmann G, Tschop M, Fischer R, et al. High altitude increases circulating interleukin-6, interleukin-1 receptor increases circulating interleukin-6, interleukin-1 receptor 52 .

14 Kato H, Yoneyama Y, Araki T. Fetal plasma lipid peroxide levels in pregnancies complicated by preeclampsia. Gynecol Obstet Invest 1997;43:158-61.

15 Poranen AK, Ekblad U, Uotila P, et al. Lipid peroxidation and antioxidants in normal and pre-eclamptic pregnancies. Placenta 1996;17:401-5.

16 Kaar K, Jouppila P, Kuikka J, et al. Intervillous blood flow in normal and complicated late pregnancy measured by means of an intravenous 133Xe method. Acta Obstet Gynecol Scand 1980;59:7-10

17 Hingorani AD, Cross J, Kharbanda RK, et al. Acute systemic inflammation impairs endothelium-dependent dilations in humans. British Hypertension Society 1999; abstract 5.1 .

18 Keogh JM, Badawi N, Kurinczuk JJ, et al. Maternal awareness of fetal seizures in pregnancies resulting in newborn encephalopathy. Acta Obstet Gynecol Scand 2000;79:787-9.

19 Steinborn A, Sohn C, Sayehli C, et al. Spontaneous labour at term is associated with fetal monocyte activation. Clin axp Immunol 1999;117:147-52.

20 Amiel-Tison C, Sureau C, Shnider SM. Cerebral handicap in full-term neonates related to the mechanical forces of labour. Baillieres Clin Obstet Gynaecol 1988;2:145-65.

21 Yagel S, Anteby E, Lavy Y, et al. Fetal middle cerebral artery blood flow during normal active labour and in labour with variable decelerations. Br f Obstet Gynaecol 1992;99:4835.

22 Wyatt JS. Cerebral oxygenation and haemodynamics in the foetus and newborn infant. Philos Trans $R$ Soc Lond B Biol Sci 1997;352:697-700.

23 Badawi N, Kurinczuk J, Keogh JM, et al. Intrapartum risk factors for newborn encephalopathy: the Western Australian case-control study. BMF 1998;317:1554-8. 